\title{
Risk-based audit selection of dairy farms
}

\author{
M. A. P. M. van Asseldonk ${ }^{\star 1}$ and A. G. J. Velthuis †‡ \\ ${ }^{*}$ Agricultural Economics Research Institute, Wageningen University and Research Centre, PO Box 35, 6706 KN Wageningen, the Netherlands \\ †Business Economics, Wageningen University, PO Box 8130, 6700 EW Wageningen, the Netherlands \\ ¥GD Animal Health Service, PO Box 9, 7400 AA Deventer, the Netherlands
}

\begin{abstract}
Dairy farms are audited in the Netherlands on numerous process standards. Each farm is audited once every 2 years. Increasing demands for cost-effectiveness in farm audits can be met by introducing risk-based principles. This implies targeting subpopulations with a higher risk of poor process standards. To select farms for an audit that present higher risks, a statistical analysis was conducted to test the relationship between the outcome of farm audits and bulk milk laboratory results before the audit. The analysis comprised 28,358 farm audits and all conducted laboratory tests of bulk milk samples 12 mo before the audit. The overall outcome of each farm audit was classified as approved or rejected. Laboratory results included somatic cell count (SCC), total bacterial count (TBC), antimicrobial drug residues (ADR), level of butyric acid spores (BAB), freezing point depression (FPD), level of free fatty acids (FFA), and cleanliness of the milk (CLN). The bulk milk laboratory results were significantly related to audit outcomes. Rejected audits are likely to occur on dairy farms with higher mean levels of SCC, TBC, ADR, and $\mathrm{BAB}$. Moreover, in a multivariable model, maxima for TBC, SCC, and FPD as well as standard deviations for TBC and FPD are risk factors for negative audit outcomes. The efficiency curve of a risk-based selection approach, on the basis of the derived regression results, dominated the current random selection approach. To capture 25,50 , or $75 \%$ of the population with poor process standards (i.e., audit outcome of rejected), respectively, only 8,20 , or $47 \%$ of the population had to be sampled based on a risk-based selection approach. Milk quality information can thus be used to preselect high-risk farms to be audited more frequently.
\end{abstract}

Key words: bulk milk, audit, sampling, monitoring

Received January 21, 2013.

Accepted October 2, 2013.

${ }^{1}$ Corresponding author: marcel.vanasseldonk@wur.nl

\section{INTRODUCTION}

Quality assurance programs or certification schemes have gained great importance in the international agribusiness sector (Albersmeier et al., 2009) and are well established within the food supply chain (Deaton, 2004; Jahn et al., 2005; Fulponi, 2006). Specific quality assurance programs or certification schemes have also been developed for dairy farms (Albersmeier et al., 2009; Velthuis and van Asseldonk, 2011). The aim of such programs is to reach a defined performance of the product by implementing process standards demanded by milk processing industries. Dairy process standards focus on multiple factors to enhance hygiene of the milking environment, hygiene of the environment in which cows are housed, hygiene of the milking equipment, udder hygiene, and cow health (Albersmeier et al., 2009). Some dairy certification schemes not only focus on farming aspects that are related to milk quality, but also assess the status of, for example, animal welfare. The certification schemes make this transparent to consumers (Herrick, 1993; Meuwissen et al., 2003). Certificates are granted and extended by means of farm audits. Farms are assessed on numerous process standards using detailed checklists. The standards include the status of farm hygiene, milking equipment, parlor, tank room, feed and water management, and veterinary medicine usage.

The traditional approach is to audit all farms within a certain time period. For example, all dairy farms in the Netherlands are audited every 2 yr. The length of the interval depends on the available audit resources. Audit resources may take the form of, for example, a constrained monetary budget or a constrained number of human-resource hours to carry out inspections.

Increasing demands for efficiency in auditing programs can be met by introducing risk-based principles. The core rationale underpinning a risk-based philosophy is that issues that present higher risks merit higher priority for resources, as these investments will yield higher cost-benefit ratios. With respect to dairy farm audits, this axiomatic foundation implies selecting subpopulations with a higher risk of poor process standards. Farms at risk should be audited more fre- 
Table 1. Summary statistics of number of audits and outcome of all audits and the audits in the analysis (i.e., audits with complete records)

\begin{tabular}{lrrrrr}
\hline & \multicolumn{2}{c}{ All audits } & & \multicolumn{2}{c}{ Audits in the analysis } \\
\cline { 2 - 3 } \cline { 5 - 6 } Outcome & \multicolumn{1}{c}{ No. } & $\%$ & & No. & $\%$ \\
\hline Approved & 60,483 & 94.18 & & 26,922 & 94.94 \\
Rejected & 3,628 & 5.64 & & 1,388 & 4.89 \\
Blocked & 113 & 0.18 & & 49 & 0.17 \\
Total & 64,222 & 100.00 & & 28,358 & 100.00 \\
\hline
\end{tabular}

quently, whereas farms with enhanced process quality control could be audited less often. The total number of selected farms to be audited and the frequency based on risk-based principles will ultimately depend on the available resources.

An essential element in a risk-based auditing approach is that prior information is available to select subpopulations of farms that are at risk. An obvious predictor is to use bulk milk quality information because bulk milk is tested regularly on several criteria. Product quality control and process quality control of bulk milk are associated (Albersmeier et al., 2009), but information about the extent of this association is too limited to set up a risk-based auditing scheme.

The goal of this study was therefore to quantify the enhanced efficiency of a risk-based sampling scheme on the basis of bulk milk quality tests compared with the traditional audit methodology of testing all farms at the same frequency. To select farms that present higher risks, a prerequisite for a risk-based sampling scheme, a statistical analysis was conducted to test the relation between the outcome of farm audits and the bulk milk test results.

\section{MATERIALS AND METHODS}

Data were provided by Qlip NV (Leusden, the Netherlands), the Dutch organization that is responsible for the certification and auditing of dairy farms and that tests all farm milk deliveries to the processors on protein, fat, lactose, urea, SCC, total bacterial count (TBC), antimicrobial drug residues (ADR), level of butyric acid spores (BAB), freezing point depression (FPD), level of FFA, and cleanliness of milk (CLN). One data set contained the results of all dairy farm audits and the other contained bulk milk laboratory results of all farm milk deliveries to the processors. The merged time-series data set of 6.5 yr included 64,222 farm audits conducted on 26,556 farms and all related laboratory results of the bulk milk samples 12 mo before the audit. In total, 28,358 complete records were included in the analysis (i.e., farm audit outcome with all laboratory outcomes before the audit).

\section{Farm Audit Data}

The outcomes of the audits were classified as approved, blocked, or rejected (Table 1). The majority of audits were classified as approved (approximately $95 \%$ ). Furthermore, a farm was blocked if no decision had been made yet for various reasons and the final decision (i.e., approved or rejected) was pending because of required additional information or actions. For this study, the $0.17 \%$ blocked farms were grouped with the rejected category because they were not directly approved. Approximately 5\% out of the 28,358 analyzed farm audits were rejected (or blocked).

Classifying farms as approved or rejected was based on a protocol containing binary checklist items and integer attention points. Each farm audit record included 271 binary checklist items that indicated a possible deviation (designated 1) from the desired farm situation (designated 0; Velthuis and van Asseldonk, 2011). For example, the checklist item "Parlor control room is dirty" has a value of 1 if the auditor observed dirt in the parlor control room, otherwise it has value 0 (which is the desired farm situation). Additionally, the data set included 52 integer variables with the number of attention points given to a specific farm category where the baseline value was 0 (Albersmeier et al., 2009). An approved farm checks almost all checklist items and has limited attention points, both indicating possible deviations from the desired farming situation. Rejected farms have important deviations in terms of failed checklist items and a high number of attention points (Velthuis and van Asseldonk, 2011).

\section{Bulk Milk Laboratory Data}

For the routine monitoring of bulk milk, a sample from each bulk milk delivery is taken and analyzed in the laboratory for composition and quality. The analysis on the composition is assessed every delivery and includes fat, protein, lactose, and urea levels. The analysis on quality includes SCC, TBC, ADR, BAB, FPD, FFA, and CLN. A description of the current test procedures applied in the Netherlands is elaborated on 
by Velthuis and van Asseldonk (2011). Test frequency differs between quality aspects, ranging from every delivery (ADR), twice a month (SCC and TBC), once a month (BAB and CLN), to twice a year (FPD and FFA). The combination of different tests and its frequency reveals product quality accurately and provides an impression of hygiene management at farm level.

Table 2 shows the descriptive statistics of the 7 laboratory quality variables $\mathrm{SCC}, \mathrm{TBC}, \mathrm{ADR}, \mathrm{BAB}$, FPD, FFA, and CLN) for the period of 12 mo before an audit. For ADR, BAB, and CLN, only the number of observations (SUM) exceeding the set threshold limits are accounted for (Albersmeier et al., 2009). The $\mathrm{ADR}$ variable deviated from the norm if residues were detected for sulfonamides, $\beta$-lactam residues (penicillin and cephalosporin), amino glycosides, macrolides, or tetracyclines. The BAB variable deviated from the norm if gas formation was observed in at least 1 of the 2 test tubes. If the sample was contaminated with, for example, sand, hair, skin flakes, or straw, CLN deviated from the norm. The average number of deviations from the norm for ADR, BAB and CLN was 0.15 (ADR SUM), 1.00 (BAB_SUM), and 0.03 (CLN_SUM), respectively, in a 12 mo period.

For SCC, TBC, FPD, and FFA, 3 descriptive statistics were included in the analysis to capture the distribution of test outcomes in the 12-mo period: the average (AVG), maximum (MAX), and standard deviation (STD). The variable SCC_AVG represents the average of the SCC results of 24 tank milk samples delivered in 12 mo before a farm audit. For SCC_AVG (1,000 cells/
$\mathrm{mL}$ ), the average was 221 . The average bacterial count for TBC_AVG $(1,000$ cells $/ \mathrm{mL})$ was 10 . The values of FPD_AVG and FFA_AVG were $521\left(\times-1 / 100^{\circ} \mathrm{C}\right)$ and $43(\mathrm{mmol} / 10,000 \mathrm{~g}$ of fat).

\section{Statistical Analysis}

A multivariable regression analysis was used to test the relation between the dependent variable $\gamma$ (i.e., outcome of forthcoming farm audit) and predictor variables (i.e., prior information with respect to the bulk milk laboratory results):

$$
\begin{aligned}
\operatorname{logit}\left(y_{a}\right) & =\beta_{0}+\beta_{1, n} \cdot \text { auditor }+\beta_{2} \cdot \mathrm{LN}\left(\mathrm{SCC}_{-} \mathrm{AVG}_{a}\right) \\
& +\beta_{3} \cdot \mathrm{LN}\left(\mathrm{SCC}_{-} \mathrm{MAX}_{a}\right)+\beta_{4} \cdot \mathrm{LN}\left(\mathrm{SCC}_{-} \mathrm{STD}_{a}\right) \\
& \left.+\beta_{5} \cdot \mathrm{LOG}\left(\mathrm{TBC} \_\mathrm{AVG}_{a}\right)+\beta_{6} \cdot \mathrm{LOG}_{(\mathrm{TBC}} \mathrm{MAX}_{a}\right) \\
& \left.+\beta_{7} \cdot \mathrm{LOG}_{(\mathrm{TBC}} \mathrm{STD}_{a}\right)+\beta_{8} \cdot \mathrm{ADR}_{-} \mathrm{SUM}_{a} \\
& +\beta_{9} \cdot \mathrm{BAB} \_\mathrm{SUM}_{a}+\beta_{10} \cdot \mathrm{FPD} \_\mathrm{AVG}_{a} \\
& +\beta_{11} \cdot \mathrm{FPD} \mathrm{MAX}_{a}+\beta_{12} \cdot \mathrm{FPD} \_\mathrm{STD}_{a} \\
& +\beta_{13} \cdot \mathrm{FFA}_{-} \mathrm{AVG}_{a}+\beta_{14} \cdot \mathrm{FFA}_{-} \mathrm{MAX}_{a} \\
& +\beta_{15} \cdot \mathrm{FFA}_{-} \mathrm{STD}_{a}+\beta_{16} \cdot \mathrm{CLN}_{-} \mathrm{SUM}_{a}+\varepsilon_{a}
\end{aligned}
$$

where $n$ represents the auditor number, $a$ the audit observation, and $\varepsilon$ the error term.

\begin{tabular}{|c|c|c|c|c|c|c|c|c|}
\hline \multirow[b]{2}{*}{ Variable $^{1}$} & \multicolumn{4}{|c|}{ All audits } & \multicolumn{4}{|c|}{ Audits in the analysis } \\
\hline & 5 th & Median & Average & 95 th & 5 th & Median & Average & 95 th \\
\hline SCC_AVG & 117.00 & 213.25 & 220.07 & 344.30 & 119.60 & 214.24 & 220.83 & 343.09 \\
\hline SCC_MAX & 168.00 & 340.00 & 362.99 & 631.00 & 184.00 & 350.00 & 374.26 & 639.00 \\
\hline SCC_STD & 22.94 & 59.35 & 66.50 & 133.35 & 27.43 & 60.74 & 67.87 & 132.29 \\
\hline TBC_AVG & 2.60 & 7.13 & 10.33 & 27.60 & 2.71 & 6.92 & 9.80 & 25.88 \\
\hline TBC_MAX & 6.00 & 18.00 & 49.59 & 173.00 & 7.00 & 19.00 & 51.44 & 180.00 \\
\hline TBC_STD & 1.38 & 4.12 & 11.36 & 39.69 & 1.42 & 4.25 & 11.25 & 39.24 \\
\hline ADR_SUM & 0.00 & 0.00 & 0.11 & 1.00 & 0.00 & 0.00 & 0.15 & 1.00 \\
\hline BAB_SUM & 0.00 & 0.00 & 0.93 & 4.00 & 0.00 & 0.00 & 1.00 & 4.00 \\
\hline FPD_AVG & 513.00 & 521.00 & 520.46 & 526.67 & 513.50 & 521.00 & 520.52 & 526.00 \\
\hline FPD_MAX & 514.00 & 522.00 & 521.63 & 528.00 & 516.00 & 523.00 & 522.51 & 528.00 \\
\hline FPD_STD & 0.00 & 2.12 & 2.54 & 6.36 & 0.00 & 2.12 & 2.51 & 6.36 \\
\hline FFA_AVG & 27.00 & 39.00 & 43.29 & 73.00 & 27.00 & 39.00 & 43.16 & 72.00 \\
\hline FFA_MAX & 29.00 & 42.00 & 47.80 & 84.00 & 29.00 & 42.00 & 47.91 & 84.00 \\
\hline FFA_STD & 1.00 & 4.00 & 6.57 & 20.00 & 1.00 & 4.00 & 6.57 & 20.00 \\
\hline CLN_SUM & 0.00 & 0.00 & 0.02 & 0.00 & 0.00 & 0.00 & 0.03 & 0.00 \\
\hline
\end{tabular}

The outcomes of the audits were classified as approved or rejected (i.e., dichotomous dependent variable). Therefore, a logistic regression model was fitted using the log-link function. The SCC and TBC variables were transformed by means of natural logarithm

Table 2. Summary statistics (5th and 95th percentiles, median, average) of the laboratory result variables of bulk milk of the total data set (n $=64,222$ audits) and of the audits used in the final model (28,358 audits)

${ }^{1}$ First 3 characters represent a milk quality criterion and the last 3 characters the summary statistic of laboratory results of the criterion during the defined period. $\mathrm{SCC}=$ somatic cell count $(\times 1,000$ cells $/ \mathrm{mL}), \mathrm{TBC}=$ total bacterial count $(\times 1,000$ cells $/ \mathrm{mL}), \mathrm{ADR}=$ presence of antimicrobial drug residues (no. in $12 \mathrm{mo}$ ), $\mathrm{BAB}=$ level of butyric acid bacteria (no. in $12 \mathrm{mo}$ ), and $\mathrm{FPD}=$ freezing point depression $\left(\times-1 / 100^{\circ} \mathrm{C}\right.$ ), FFA $=$ level of free fatty acid $(\mathrm{mmol} / 10,000 \mathrm{~g}$ of fat), and CLN = cleanliness of the milk (no. in $12 \mathrm{mo})$. AVG $=$ average, STD $=$ standard deviation, MAX = maximum, and SUM = number of times that the attention limit is exceeded. 
Table 3. Odds ratios (OR) and $95 \%$ confidence intervals of OR

\begin{tabular}{|c|c|c|c|}
\hline Variable $^{1}$ & OR & $\begin{array}{l}\text { Lower } 95 \% \\
\text { bound OR }\end{array}$ & $\begin{array}{l}\text { Upper } 95 \% \\
\text { bound OR }\end{array}$ \\
\hline LN(SCC_AVG) & 3.142 & 2.237 & 4.413 \\
\hline LN(SCC_MAX) & 0.582 & 0.441 & 0.767 \\
\hline LOG(TBC_AVG) & 13.064 & 8.556 & 19.947 \\
\hline LOG(TBC_MAX) & 3.194 & 1.555 & 6.560 \\
\hline LOG(TBC_STD) & 0.148 & 0.076 & 0.287 \\
\hline ADR SUM & 1.180 & 1.094 & 1.272 \\
\hline BAB_SUM & 1.309 & 1.129 & 1.518 \\
\hline FPD_MAX & 0.984 & 0.973 & 0.995 \\
\hline FPD_STD & 1.020 & 1.004 & 1.036 \\
\hline
\end{tabular}

${ }^{1}$ First 3 characters represent a milk quality criterion and the last 3 characters the summary statistic of laboratory results of the criterion during the defined period. SCC $=$ somatic cell count $(\times 1,000$ cells $/ \mathrm{mL})$, TBC = total bacterial count $(\times 1,000$ cells $/ \mathrm{mL}), \mathrm{ADR}=$ presence of antimicrobial drug residues (no. in $12 \mathrm{mo}), \mathrm{BAB}$ $=$ level of butyric acid bacteria (no. in $12 \mathrm{mo})$, and $\mathrm{FPD}=$ freezing point depression $\left(\times-1 / 100^{\circ} \mathrm{C}\right)$. AVG $=$ average, $\mathrm{STD}=$ standard deviation, $\mathrm{MAX}=$ maximum, and $\mathrm{SUM}=$ number of times that the attention limit is exceeded.

and logarithm, respectively (Velthuis and van Asseldonk, 2011). The auditor $(\mathrm{n}=47)$ was included in the analysis for statistical correction.

First, all bulk milk laboratory variables (as listed in Table 2) were tested in a univariable analyses and ranked based on their $P$-value. The bulk milk laboratory variables with a $P$-value $>0.25$ were excluded from the multivariable analyses. Second, all selected bulk milk laboratory variables were included in the model as ranked based on the $P$-value in the univariable analyses. Then, variables with highest $P$-values, where $P>$ 0.05 , were removed one by one until all variables in the model met the criterion of $P$-value $<0.05$ (backward procedure of GenStat, 11th ed.; VSNi, 2009). Goodness of fit of the models was determined by the $\mathrm{R}^{2}$ values for the model (Nagelkerke $R^{2}$ ) and by means of the classification statistics (i.e., percentage correctly classified as being approved or rejected). Multi-collinearity checks were performed and variables causing multi-collinearity were dropped from the final model. Regression coefficients are used to estimate odds ratios $[\exp (\beta)]$ and the corresponding confidence interval for each of the independent variables in the model.

The standard cut-off value of 0.5 was used in the regression model. Scores higher than this cut-off were predicted as approved and scores lower as rejected. The cut-off value was adjusted for a better predictive fit given the specific context (i.e., selection of farms at risk to be rejected). A series of cut-off values ranging from 0.005 to 0.50 was analyzed. Finally, the efficiency curve of a risk-based approach, based on classification statistics, versus random selection was plotted.

\section{RESULTS}

Nine bulk milk laboratory variables were significantly related to the audit outcomes in the final model. Mean or sum levels of SCC, TBC, ADR, and BAB affected the probability that a forthcoming audit would be rejected. Moreover, the recorded maxima for SCC, TBC, and FPD, and standard deviations for TBC and FPD were also risk factors. In the univariable analysis, 11 of the 15 were statistically significant.

The strength of association between bulk milk laboratory variables and audit outcome was estimated by means of odds ratios and are shown in Table 3 (as well as corresponding confidence intervals of the model). For example, from the odds ratio of 3.14 for $\mathrm{LN}\left(\mathrm{SCC}_{-}\right.$ AVG), we can conclude that an audit outcome of being rejected is 3.14 times more likely for a dairy farm with a 1-point increase in $\mathrm{LN}\left(\mathrm{SCC} \_\mathrm{AVG}\right)$ during the 12-mo period before the audit compared with the mean of all dairy farms (i.e., 221,000 cells $/ \mathrm{mL}$ ). This 3.14 equals $1,000 \times \mathrm{e}^{3.14}=23,104$ cells $/ \mathrm{mL}$ (with a confidence interval ranging from 9,207 to 84,774 cells $/ \mathrm{mL}$ ).

Of the 9 significant bulk milk laboratory variables, 6 odds ratios were greater than 1, indicating that the probability of a rejected audit is more likely to occur at elevated levels, whereas elevated levels of LN(SCC_MAX), LOG(TBC_STD), and FPD_MAX reduced the risk of a rejected audit. Note that for each of these laboratory test variables, other terms of the same laboratory tests were included in the model (i.e., AVG, MAX, or STD). In case of SCC, the average was included, for TBC the average as well as maximum values, and for FPD the maximum value was included.

The $\mathrm{R}^{2}$ of the final model was 0.13 (Nagelkerke). Rather than this goodness of fit statistic, the proportion of cases classified correctly was of interest. The classification table (Table 4) shows the classification statistics with alternative cut-off values. Given a cutoff value of 0.50 , almost $100 \%$ of farms audited were correctly classified for the "to be accepted" group and merely $1.60 \%$ to the "to be rejected" group. Overall, 
Table 4. Classification table with alternative cut-off values

\begin{tabular}{lccc}
\hline & \multicolumn{2}{c}{ Percentage correct } & \\
\cline { 2 - 3 } $\begin{array}{l}\text { Cut-off } \\
\text { value }\end{array}$ & Approved & Rejected & Total \\
\hline 0.5 & 100 & 1.6 & 95.0 \\
0.4 & 99.9 & 2.9 & 94.9 \\
0.3 & 99.5 & 5.6 & 94.8 \\
0.2 & 98.5 & 11.6 & 94.1 \\
0.1 & 90.2 & 36.6 & 87.4 \\
0.09 & 87.6 & 42.2 & 85.3 \\
0.08 & 84.3 & 48.4 & 82.5 \\
0.07 & 79.8 & 55.1 & 78.6 \\
0.065 & 77.0 & 58.8 & 76.0 \\
0.06 & 74 & 62.1 & 73.4 \\
0.05 & 66.6 & 68.5 & 66.7 \\
0.045 & 62.4 & 72.3 & 62.9 \\
0.04 & 57.2 & 76.6 & 58.2 \\
0.035 & 51.5 & 81.7 & 53.1 \\
0.03 & 45.1 & 86.4 & 47.1 \\
0.025 & 37.4 & 90.3 & 40.1 \\
0.02 & 29.1 & 93.7 & 32.4 \\
0.015 & 19.8 & 96.8 & 23.7 \\
0.01 & 10 & 98.7 & 14.5 \\
0.005 & 2.6 & 99.7 & 7.5 \\
\hline
\end{tabular}

94.98\% of farms audited were correctly classified. At lower cut-off values, smaller deviations in milk laboratory results before the audit would lead more quickly to a classification into the "to be rejected" group. In summary, the classification statistic for the "to be rejected" group improved considerably at lower cut-off levels at the expense of the classification statistic of the "to be accepted" group.

Based on the derived regression results, the efficiency curve of a risk-based approach was plotted (Figure 1). For a given percentage of dairy farms to be enrolled on risk-based principles for auditing, the corresponding percentage of farms with actual percentage of rejections is depicted.

The efficiency curve of a risk-based approach dominated the random selection approach. The convex efficiency curve was lower compared with the random selection line of $\mathrm{x}=\mathrm{y}$. For example, to capture 25, 50, or $75 \%$ of the population with poor process standards (i.e., rejected audit), respectively, only 8,20 , or $47 \%$ of the population had to be sampled based on a risk-based approach. The difference in the percentage of farms to be audited risk-based versus a random approach in percentage points was $17 \%$ (25-8\%), 30\% (50-20\%), and $28 \%(75-47 \%)$ respectively. These results imply that 3.13 times fewer dairy farms $(28 \% / 8 \%), 2.50$ times fewer dairy farms $(50 \% / 20 \%)$, and 1.60 times fewer dairy farms $(75 \% / 48 \%)$ could be audited while obtaining the same level of process quality standards (if the objective function was to audit 25,50 , or $75 \%$ of farms at risk, respectively). Thus, the relative advantage of a risk-based approach is more substantial if the objective is to trace a smaller fraction of dairy farms that are more likely to have a future rejected audit. In summary, based on this study, milk quality information can be used to preselect farms that should be audited more frequently.

\section{DISCUSSION}

This study is unique, because the 6.5 yr time-series data set contained extensive information on farm audits as well as 7 different laboratory tests of bulk milk samples. In addition, the protocol of classifying audits as approved or rejected has evolved over time to include extensive lists with checklist items. To investigate the effect of the time period analyzed, with a gradually evolving protocol and checklist, a robustness check was applied. Regression model results for the whole 6.5-yr period were compared with results obtained from those estimated from observations of the most recent $2.5 \mathrm{yr}$. Bulk milk test variables included and odds ratios differed in both models to discriminate between accepted and rejected audits. In general, rejected audits were more likely to occur on dairy farms with higher mean levels of SCC, TBC, ADR, and CLN. Moreover, higher maxima for FPD and elevated standard deviations for TBC were common risk factors. Overall, both models included the majority of significant bulk milk test variables and therefore had a good robustness, at least for the Netherlands. Nevertheless, in other countries, with other bulk milk test procedures and other audit checklists, a country-specific analysis is required.

In the current research, aggregated statistics for the laboratory tests were based on a 12 -mo period before the audit. This 12-mo period was chosen for several

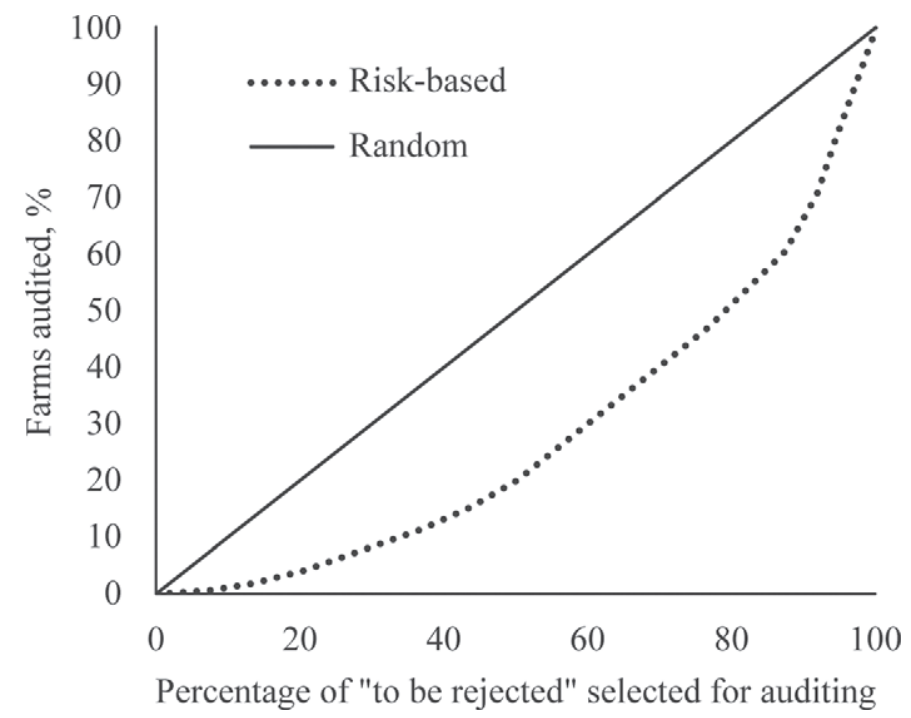

Figure 1. Efficiency curve of a risk-based approach versus random selection to select dairy farms for a farm audit. 
reasons. The pragmatic justification is that some of the tests were conducted only twice a year (FPD and FFA). A more fundamental justification is that audits are conducted to reach a defined and stable performance of the production process. Short-term laboratory test statistics (e.g., based on a 2-mo period) would pick up short-term deviations as a result of operational problems, whereas long-term laboratory test statistics would pick up tactical problems. Additional statistical analysis with respect to the time period (i.e., 2, 6, or $12 \mathrm{mo}$ ) revealed high correlations among the averages, although levels differed. For example, SCC_AVG for 12,6 , or 2 mo was $67,000,61,000$, and 52,000 cells $/ \mathrm{mL}$, respectively. Standard deviations and maximum values were more dependent on the period accounted for. Long-term statistics were more discriminatory in the model and increased the amount of variance explained by the model than did the short-term statistics.

The risk-based audit approach focused solely on targeting subpopulations with higher risk of poor process standards compared with the population as a whole. A complementary approach is to reconsider the audit checklist elements based on prior information obtained from laboratory test results. In general, the purpose is to align the audit with the risk situation and risk potential of the individual firm (Albersmeier et al., 2010). This might result in more customized, risk-based audits: shorter audits for selected dairy farms and longer or specific audits for other dairy farms. The focus is to limit the audit risk; that is, when the audit certificate is granted even though the firm has significant deficiencies (Albersmeier et al., 2010).

\section{CONCLUSIONS}

Quality control of bulk milk is a predictor for process quality control. The efficiency curve of a risk-based approach, on basis of the derived regression results, dominated a random selection approach. To capture 25, 50, or $75 \%$ of the population with poor process standards (i.e., audit outcome of rejected), only 8, 20, or $47 \%$, respectively, of the population had to be sampled based on a risk-based approach. Milk quality information can thus be used to preselect farms that should be audited more frequently. Therefore, a risk-based surveillance system that includes subpopulations at higher risk can be designed, which provides an improved process quality control compared with a random sample design.

\section{ACKNOWLEDGMENTS}

The authors express their gratitude to Sector Boerderijmelk, Qlip NV (Leusden, the Netherlands; Louwrens van Keulen) for funding this study and for providing data, and to Johan Tippe (Qlip NV) for his contributions to interpretation of data, results, and discussion.

\section{REFERENCES}

Albersmeier, F., H. Schulze, G. Jahn, and A. Spiller. 2009. The reliability of third-party certification in the food chain: From checklists to risk-oriented auditing. Food Contr. 20:927-935.

Albersmeier, F., H. Schulze, and A. Spiller. 2010. System dynamics in food quality certifications: Development of an audit integrity system. Int. J. Food System Dynamics 1:69-81.

Deaton, B. J. 2004. A theoretical framework for examining the role of third-party certifiers. Food Contr. 15:615-619.

Fulponi, L. 2006. Private voluntary standards in the food system: The perspective of major food retailers in OECD countries. Food Policy 31:1-13.

Herrick, J. B. 1993. Milk and dairy beef quality assurance programs: A food safety issue. J. Am. Vet. Med. Assoc. 203:1389-1399.

Jahn, G., M. Schramm, and A. Spiller. 2005. The reliability of certification: Quality labels as a consumer policy tool. J. Consum. Policy 28:53-73.

Meuwissen, M. P. M., A. G. J. Velthuis, H. Hogeveen, and R. B. M. Huirne. 2003. Traceability and certification in meat supply chains. J. Agribus. 21:167-181.

Velthuis, A. G. J., and M. A. P. M. van Asseldonk. 2011. Process audits versus product quality monitoring of bulk milk. J. Dairy Sci. 94:235-249.

VSNi. 2009. GenStat. 11th ed. Accessed Nov. 20, 2013. http://www. vsni.co.uk/. 